\title{
Model Based Anticontrol of Chaos
}

\author{
Ömer Morgül \\ Bilkent University \\ Dept. of Electrical and Electronics Engineering \\ 06533, Bilkent, Ankara, Turkey \\ fax : 90-312-266 4192 \\ e-mail : morgul@ee.bilkent.edu.tr
}

\begin{abstract}
We will consider model based anticontrol of chaotic systems. We consider both continuous and discrete time cases. We first assume that the systems to be controlled are linear and time invariant. Under controllability assumption, we transform these systems into some canonical forms. We assume the existence of chaotic systems which has similar forms. Then by using appropriate inputs, we match the dynamics of the systems to be controlled and the model chaotic systems.
\end{abstract}

\section{Introduction}

The analysis and control of chaotic behaviour in dy. namical systerns has been investigated by many researchers in various disciplines on recent years. The literature is quite rich on the subject, and interested reader may consult to e.g. [2],[3], [4], [8], ,9].

The seminal work of $[15]$ motivated the research in the field of chaos control, and the term "controlling chaos" was introduced in the literature. In most of the works in the area of chaos control the main aim is the suppression of chaotic behaviour, see e.g. [4], [9]. On the other hand, the opposite aim i.e. to retain the chaotic behaviour, or even to force a regular behaviour into a chaotic one, is also an interesting problern and received attention by many researchers. This problem may be called as "anticontrol", see e.g. [16], or as "chaotification", see e.g. [18]. Apparently, this scheme has many potential applications in many fields, see e.g. $[1],[5],[6][7]$.

In this work, we will consider a model-based approach to the anticontrol problem. We consider both continuous and discrete time cases for the systems to be controlled. We first assume that the systerns to be controlled are linear and time invariant. By assuming the controllability, we first transform these systerns into some appropriate form. We assume the existence of chaotic model systems in a similar form. Then we try to match the dynamics of the system to be controlled with that of the model chaotic system by means of an appropriate control input. We prove that:

$i$ : any controllable linear time-invariant system can be chaotized with an appropriate input,

$i i$ : this approach could be generalized to a class of nonlinear systems.

Since our approach relies on the existence of chaotic models in an appropriate form, whether there exist such models in arbitrary dimensions is a relevant question. We propose a simple procedure to generate such chaotic models in arbitrary dimensions. Another question we consider is the computability of the required feedback law by using only the available signals. To estimate the states of the system to be controlled, we propose an observer-based synchronization scheme. Under some mild conditions, exponentially fast synchronization may be achieved, and one can use the estimated states to compute the feedback law.

This paper is organized as follows. In the next section we give the problems considered in this paper, considering both continuous and discrete time cases. In the following sections, we propose solution schemes for both cases. Then we present simple schemes to generate model chaotic systems. Finally we give some concluding remarks.

\section{Problem Statement}

We will first consider the linear systems. We assume that the system to be controlled is given in continuous time case as

$$
\dot{x}=A x+B u, \quad y=C x
$$


and in the discrete time case as follows

$$
x(k+1)=A x(k)+B u(k), \quad y(k)=C x(k),
$$

where $x \in \mathbf{R}^{n}, A \in \mathbf{R}^{n \times n}$ is a constant matrix, $B, C^{T} \in \mathbf{R}^{n}$ are constant vectors, here superscript $T$ denotes transpose, $u$ is the (scalar) control input and $y$ is the (scalar) output, which is assumed to be measurable, and the discrete time index $k=0,1,2, \ldots$ is an integor. For this system, we pose the following problems :

Problem 1 : Find a feedback law $u=g(x)$, where $g: \mathbf{R}^{n} \rightarrow \mathbf{R}$ is an appropriate function, such that the resulting closed-loop system exhibits chaotic behaviour.

Problem 2: Assume that the feedback law $u=$ $g(x)$, which solves problem 1 , cannot be computed by using the output $y$ alone. Find an approximate control law $u=\hat{u}$, which can be computed by using oltput, such that $\|\hat{u}-g(x)\| \rightarrow 0$ as $t \rightarrow \infty$ in the continuous time case and as $k \rightarrow \infty$ in the discrete time case; here $x$ is the solution of (1) or (2), and $\|\cdot\|$ denotes any norm in $\mathbf{R}^{n}$.

A solution to problem 1 will be provided in the next section. Later we will present an observer based scheme for problem 2 . In this approach, the output $y$ will be used as a synchronization signal, and an observer based synchronization scheme will be used to estimate the states $x$ of (1) or (2), see e.g. [12], [13]. 'These estimates then will be used to obtain an approximation of the control law $u=g(x)$.

To simplify the analysis, we will first transform the system given by (1) and (2) into an appropriate canonical form. Let us define the following matrix

$$
Q_{c}=\left(\begin{array}{lll}
A^{n-1} B & A^{n-2} B \cdots A B B
\end{array}\right) .
$$

It is well-known that the system given by (1) or (2) is controllable (i.e. any state $x_{0} \in \mathbf{R}^{n}$ can be steered to any state $x_{1} \in \mathbf{R}^{n}$ with an appropriate control input $u$ ) if and only if $\operatorname{rank}\left(Q_{c}\right)=n$, see e.g. [11]. We will assume that the systems given by (1) or (2) are controllable, hence $Q_{c}$ is invertible.

Let $p(\lambda)$ be the characteristic polynomial of $A$ given by (1) or (2), as follows:

$p(\lambda)=\operatorname{det}(\lambda I-A)=\lambda^{n}+\alpha_{1} \lambda^{n-1}+\ldots+\alpha_{n-1} \lambda+\alpha_{n}$.

Now, let: us define the vectors $u_{1}=\left(\begin{array}{ll}1 & \alpha_{1} \ldots \alpha_{n-1}\end{array}\right)^{T^{\prime}}$, $u_{2}=\left(\begin{array}{lllll}0 & 1 & \alpha_{1} & \ldots \alpha_{n-2}\end{array}\right)^{T}, . ., u_{n}=\left(\begin{array}{lll}0 & 0 & \ldots\end{array}\right)^{T}$, and define the matrices $U=\left(u_{1} u_{2} \ldots u_{n}\right), R=\left(Q_{c} U\right)^{-1}$. By using the coordinate transformation $z=R x$, (1) and (2) can be transformed into the following form, respectively :

$$
\begin{gathered}
\dot{z}=\hat{A} z+\hat{B} u \quad, \quad y=\hat{C} z \\
z(k+1)=\hat{A} z(k)+\hat{B} u(k) \quad, \quad y=\hat{C} z
\end{gathered}
$$

where $z=\left(\begin{array}{llll}z_{1} & z_{2} & \ldots & z_{n}\end{array}\right)^{T}, \hat{A}=R A R^{-1}, \hat{B}=R B$, $\hat{C}=C R^{-1}$. After straightforward calculations and by using Cayley-Hamilton theorem (i.e. $p(A)=0$, where $p(\cdot)$ is given by (4)), it can be shown that $\hat{A}$ and $\hat{B}$ have the following form :

$$
\begin{gathered}
\hat{A}=\left(\begin{array}{ccccc}
0 & 1 & 0 & \ldots & 0 \\
0 & 0 & 1 & \ldots & 0 \\
& & \vdots & & \\
0 & 0 & 0 & \ldots & 1 \\
-\alpha_{n} & -\alpha_{n-1} & -\alpha_{n-2} & \ldots & -\alpha_{1}
\end{array}\right) \\
B=\left(\begin{array}{lllll}
0 & 0 & 0 & 0 & 1
\end{array}\right)^{T}
\end{gathered}
$$

\section{An Anti-Control Scheme}

We assume the existence of a chaotic system which has the following form in the continuous time case ( for $n \geq 3$ )

$$
\left.\begin{array}{c}
\dot{w}_{1}=w_{2} \\
\dot{w}_{2}=w_{3} \\
\vdots \\
\dot{w}_{n-1}=w_{n} \\
\dot{w}_{n}=f\left(w_{1}, w_{2}, \ldots, w_{n}\right)
\end{array}\right\}
$$

and the following form in the discrete time case :

$$
\left.\begin{array}{c}
w_{1}(k+1)=w_{2}(k) \\
w_{2}(k+1)=w_{3}(k) \\
\vdots \\
w_{n-1}(k+1)=w_{n}(k) \\
w_{n}(k+1)=f\left(w_{1}(k), w_{2}(k), \ldots, w_{n}(k)\right)
\end{array}\right\}
$$

where $f: \mathbf{R}^{n} \rightarrow \mathbf{R}$ is an appropriate function. In the continuous time case, for $n=3$ there are many chaotic systems proposed in the literature which has the form given above, see e.g. [12], [13]. In fact, many chaotic electronic oscillators proposed in the literature, including the well-known Chua's oscillator, are either in this form, or could be transformed into this form. For the discrete time case, for $n=1$, the system given by (10) reduces to $w(k+1)=f(w(k))$, and there are many one dimen sional chaotic systems which has this form, e.g. logistic equation. For $n=2$, the well-known Hénon system can be easily transformed into this form. Later we will present a simple scheme to generate such model chaotic systems. 
Our anti-control scheme is based on matching the system given by (5) and (6) with the model chaotic system given by (9) and (2), respectively, by using an appropriate control input. $u$. Note that (9) could be rewritten as

$$
\dot{w}=\hat{A} w+\hat{B} h(w)
$$

where $w=\left(\begin{array}{llll}w_{1} & w_{2} & \ldots & w_{n}\end{array}\right)^{T}$, and

$$
h(w):=f(w)+\alpha_{1} w_{n}+\alpha_{2} w_{n-1}+\ldots+\alpha_{n} w_{1}
$$

Similarly, (10) could be rewritten as

$$
w(k+1)=\hat{A} u(k)+\hat{B} h(w(k)),
$$

where $u==\left(\begin{array}{llll}u_{1} & w_{2} & \ldots & w_{n}\end{array}\right)^{T}$, and

$$
\begin{gathered}
h(u(k))=f(u(k))+\alpha_{1} w_{n}(k)+\alpha_{2} u w_{n-1}(k) \\
+\ldots+\alpha_{n} w_{1}(k)
\end{gathered}
$$

Here, $\alpha_{;}$are arbitrary constants.

To achieve the matching between the model and the system to be controlled, we can choose the control inputi as .

$$
u=h(z)=f(z)+\alpha_{1} z_{n}+\alpha_{2} z_{n-1}+\ldots+\alpha_{n} z_{1},
$$

to transform (5) into the chaotic system given by (9). Similarly, in the discrete time case we may choose the control input as :

$$
\begin{gathered}
u(k)=h(z(k))=f(z(k))+\alpha_{1} z_{n}(k)+\alpha_{2} z_{n-1}(k), \\
+\ldots+\alpha_{n} z_{1}(k)
\end{gathered},
$$

to traisform (6) into the chaotic system given by (10)

The approach given above can also be applied to a class of nonlinear systems. Let us assume that the system to be controlled is given as :

$$
i=A(x)+B(x) u \quad, \quad y=C(x) \quad,
$$

in the continuous time case and as

$$
x(k+1)=A(x(k))+B(x(k)) u(k), y(k)=C(x(k))
$$

in the discrete time case, where $A ; B: \mathbf{R}^{n} \rightarrow \mathbf{R}^{n}$ and $C: \mathbf{R}^{n} \rightarrow \mathbf{R}$ are appropriate functions, $u$ and $y$ are control input and measurement outputs, respectively. which are scalars.

Wo assume that there exists a coordinate change $z=T(x)$, where $T: \mathbf{R}^{n} \rightarrow \mathbf{R}^{n}$ is an appropriate function, which transforms (17) into the following form.

$$
\dot{z}=\hat{A} z+\hat{B}(\gamma(z)+\dot{\beta}(z) u) \quad, \quad y=\hat{C}(z)
$$

and (18) into the following form :

$$
\begin{gathered}
z(k+1)=\hat{A} z(k)+\hat{B}(\gamma(z(k))+\beta(z(k)) u(k)), \\
y(k)=\hat{C}(z(k)),
\end{gathered}
$$

where $\hat{A}, \hat{B}$ are as given in (7) and (8), $\alpha_{i}, i=$ $1, \ldots, n$ are appropriate constants, and $\gamma, \beta, \hat{C}$ : $\mathbf{R}^{n} \rightarrow \mathbf{R}$ are appropriate functions. Note that the terms multiplying $\alpha_{i}$ in (20) could be included in $\gamma(\cdot)$.

In the continuous time case, by using the control law

$$
\imath=\frac{h(z)-\gamma(z)}{\beta(z)},
$$

where $h(\cdot)$ is given by $(12)$, we can match the dynamics of (19) with that of the model chaotic system given by (11), provided that $\beta(z) \neq 0$. This requirement is natural, since otherwise the control input $u$ has no effect on the system dynamics, see (19).

Similarly, in the discrete time case, an appropriate control input $u(k)$ to obtain a model match between $(20)$ and $(10)$ is given as follows :

$$
u(k)=\frac{h(z(k))-\gamma(z(k))}{\beta(z(k))},
$$

where $h($.$) is given by (14). Obviously, we require$ $\beta(z(k)) \neq 0$ along the solutions of $(20)$. This requirement is natural, since otherwise the control input $u(k)$ has no effect on the system dynamics, see $(20)$.

The results presented in this section can be summarized as follows

i : Any controllable linear (single input) system can be chaotified with an appropriate control law.

ii : Any nonlinear (single input) system which could be transformed into the form (19) or (20) can be chaotified with an appropriate control law provided that $\beta(z(k)) \neq 0$.

\section{Synchronization Based Implementation}

To implement the control laws given above the state vector $z$ should be available. In most of the cases, the available output signal $y$ has lower dimension, which is a scalar in our case, and is not sufficient to compute the necessary control input $u$. In such cases, an appropriate approach would be to obtain an approximation $\hat{z}$ of $z$, and use this estimate to approximate the required control signal.

Since the synchronization schemes may provide good estimates of the receiver states, which is $z$ in our case, 
a natiral approach to solve the problem 2 given in section 2 is to use a synchronization scheme for the system to be controlled. For this aim, any synchronization scheme which uses the output $y$ as a synchronization signal and provides estimates $\hat{z}$ of $z$ could be used.

'To elaborate further, let us consider the linear system given by (5). Let us consider the following observerbased synchronization scheme for the system given by (5):

$$
\hat{\hat{B}}:=\hat{A} \hat{\tilde{z}}+\hat{B} u+K(y-\hat{y}) \quad, \quad \hat{y}=\hat{C} \hat{z}
$$

where $\hat{z} \in \mathbf{R}^{n}, K \in \mathbf{R}^{n}$ is a gain vector to be determined. Let us define the error in synchronization as $\because=z-\hat{z}$. By using (5) and (24) we obtain :

$$
\dot{e}=(\hat{A}-K \hat{C}) e \text {. }
$$

Hence, if $A_{c}=\hat{A}-K \hat{C}$ is a stable matrix, then we have $\|e(t)\| \rightarrow 0$ as $t \rightarrow \infty$; moreover this decay is exponential. Existence of such a vector $K$ is guaranteed if the system given by (5) is observable. More prexisely, let us define the following observability matrix :

$$
Q_{o}=\left(\begin{array}{c}
C \\
C A \\
\vdots \\
C A^{n-1}
\end{array}\right) \text {. }
$$

It is well-known that if $\operatorname{rank}\left(Q_{0}\right)=n$, then there exists a $K$ such that the matrix $A_{c}$ is stable, hence the solutions of (25) satisfy:

$$
\|e(t)\| \leq M e^{-\delta t}\|e(0)\|
$$

for some $M>0, \delta>0$, for details see e.g. [12], [13].

Based on the estimate $\hat{z}$ of $z$, a natural approximation of $u$ given by (15) is $u=h(\hat{z})$. To see the effect of this approximation, assume that $h: \mathbf{R}^{n} \rightarrow \mathbf{R}$ is a Lipschitz function, i.e. the following holds for some $1>0$ :

$$
\|h(z)-h(\hat{z})\| \leq l\|z-\hat{z}\| .
$$

Now, assume that we use $u=h(\hat{z})$ in (5). Then, the latter becomes:

$$
\dot{\hat{z}}=\hat{A} z+\hat{B} h(\hat{z})=\hat{A} z+\hat{B} h(z)+e_{c}(t),
$$

where $e_{c}(t)$ is an error term which satisfies:

$$
\left\|e_{c}(t)\right\|=\|\hat{B}(h(\hat{z})-h(z))\| \leq l M e^{-\delta t}\|e(0)\|,
$$

see $(27),(28)$. Since the error term decays to zero exponentially fast, we expect that the behaviour of (29) and (11) be qualitatively similar, provided that the chatic behaviour of (11) is structurally stable. If the chaotic: solution of (11) is globally attractive, then since $e_{c}(t)$ decays to zero exponentially fast, the solutions of (29) will eventually converge to the chaotic solutions of (11). If the chaotic solutions of (11) are only locally attractive, let us assume that for some $\epsilon>0$, the behaviours of (29) and (11) are qualitatively similar, provided that $\left\|e_{c}(t)\right\| \leq \epsilon$. We will call this assumption as the structural stability assumption, see e.g. [8]. From (30) it easily follows that this condition is satisfied for $\|e(0)\| \leq \epsilon / l M$. Hence, if initial error is sufficiently small, then the behaviours of (29) and (11) are qualitatively similar under the structural stability assumption given above. On the other hand, assume that $\|e(0)\| \leq R$ for some $R>0$. From (30) it follows that $\left\|e_{c}(t)\right\| \leq \epsilon$ for $t \geq T=1 / \delta \ln (l M R / \epsilon)$. Hence we could use a switching law to generate $u$ as follows :

$$
u=\left\{\begin{array}{cc}
0 & t<T \\
h(\hat{z}) & t \geq T
\end{array}\right.
$$

The same approach could be generalized to the discrete time case as well. There are many such schemes proposed in the literature, see e.g. [17]. For illustrative purposes, we will consider the following observer based synchronization scheme

$$
\begin{gathered}
\hat{z}(k+1)=\hat{A} \hat{z}(k)+\hat{B} u(k)+K(y(k)-\hat{y}(k)), \\
\hat{y}(k)=\hat{C} \hat{z}(k),
\end{gathered}
$$

where $K \in \mathbf{R}^{n}$ is a gain vector to be determined. Let the synchronization error be defined as $e(k)=$ $z(k)-\hat{z}(k)$. By using $(6)$ and (32) we obtain :

$$
e(k+1)=(\hat{A}-K \hat{C}) e(k)
$$

Therefore $e(k) \rightarrow 0$ as $k \rightarrow \infty$ if and only if the matrix $A_{c}=\hat{A}-K \hat{C}$ is Schur stable (i.e. any eigenvalue $\lambda$ of $A_{c}$ satisfies $|\lambda|<1$ ). Moreover, in this case the decay is exponential, i.e. the following holds for some $M>0$ and $0<\rho<1$ :

$$
\|e(k)\| \leq M \rho^{k}\|e(0)\| .
$$

It is known that there exists such a gain vector $K$ which makes $A_{c}$ Schur stable if the system given by (2), or equivalently the system given by (6), is observable, see e.g. [11]. It is also known that the latter condition is satisfied if and only if the observability matrix $Q_{0}$ given by (26) has full rank. The same structural stability arguments presented above applies to this case as well. In particular, assume that for some $\epsilon>0$, the behaviours of (13) and (20) are qualitatively similar provided that $\left\|e_{c}(k)\right\| \leq \epsilon$, see e.g. [8], where $e_{c}$ is an error term similar to (30). It can be easily shown that this condition holds for $\|e(0)\| \leq \epsilon / c M$. Therefore, if the initial error is sufficiently small then the solutions of $(20)$ will be 
chantic provided that the chantic attractor of (13) is locally attractive and structurally stable in the sense given above. On the other hand, if $\|e(0)\| \leq R$. for some $R>0$, it can be shown that $\left\|e_{c}(k)\right\| \leq \epsilon$ for $h:>N=(\ln \epsilon-\ln c M R) / \ln \rho$. Hence we could use a switching law to generate $u$ as follows :

$$
u(k)==\left\{\begin{array}{cc}
0 & k<N \\
h(\hat{z}(k)) & k \geq N
\end{array}\right.
$$

\section{Model Chaotic Systems}

Our control scheme is based on the existence of model chaotic. systems which has an appropriate form. In this chapter, we will propose a simple scheme to generate such model chaotic systems both for continuous and discrete time cases.

First let us consider the continuous time case. For $n=3$, such chaotic systems are abundant in the litcrature. In fact, all Lur'e type systems, which cover most of the clectronic chaotic oscillators proposed in the literature including the well-known Chua's oscillator, calz be transformed into this form. Some systems, which are not in this structure (e.g. Rössler system), may be transformed into this form, see e.g. [13]. As an example, consider the following system

$$
\left.\begin{array}{c}
\dot{w}_{1}=w_{2} \\
\dot{w}_{2}=w_{3} \\
\dot{w}_{3}=-b_{2} w_{3}-b_{1} w_{2}-b_{0} w_{1}-w_{1}^{2}
\end{array}\right\} .
$$

This system exhibits chaotic behaviour for certain range of parameters $b_{i}$, see [10], [14]. To generate chrotic systems for $n>3$ which has the form of (9), let us consider the case $n=3$, which is repeated below for convenience :

$$
\left.\begin{array}{c}
\dot{w}_{1}=w_{2} \\
\dot{w}_{2}=w_{3} \\
\dot{w}_{3}=f\left(u_{1}, w_{2}, w_{3}\right)
\end{array}\right\}
$$

By defining $w=w_{1}$, and noting that $w_{2}=\dot{w}, w_{3}=$ $\ddot{u}$, and by using (38), we obtain the following scalar equation:

$$
\dot{w}^{(3)}-f(w, \dot{w}, \ddot{w})=0 .
$$

Obviously, (39) and (38) are equivalent through the transformation given above. Now let us consider the following higher dimensional siystem :

$$
\left.\begin{array}{c}
\dot{w}_{1}=w_{2} \\
\dot{w}_{2}=w_{3} \\
\dot{w}_{3}=f\left(w_{1}, w_{2}, w_{3}\right)+w_{4} \\
\dot{w}_{4}=-\alpha w_{4}
\end{array}\right\}
$$

where $\alpha>0$ is an arbitrary constant. Note that $u_{4}(t)=w_{4}(0) e^{-\tau t} \rightarrow 0$ as $t \rightarrow \infty$. Hence asymptotically, (38) and the first 3 equations of (40) are the same. Therefore, if (38) has a globally attractive chaotic solution, so does (40). On the other hand, if (38) has only locally attractive chaotic solution, which is structurally stable in the sense given before, then so does (40), provided that $\left|w_{4}(0)\right|$ is sufficiently small.

To transform the system given by (40) into the form given by (9), first note that from the third equation in (40) we have $w_{4}=\dot{w}_{3}-f\left(w_{1}, w_{2}, w_{3}\right)$. By defining $w=w_{1}$, and noting that $w_{2}=\dot{w}, w_{3}=\ddot{w}$, and using the last equation in (40) we obtain :

$$
\frac{d}{d t}\left(w^{(3)}-f(w, \dot{w}, \ddot{w})\right)+\alpha\left(w^{(3)}-f(w, \dot{w}, \ddot{w})\right)=0
$$

which could be rewritten as

$$
w^{(4)}=F\left(w, \dot{w}, \ddot{w}, w^{(3)}\right),
$$

where

$$
\begin{aligned}
F\left(w, \dot{w}, \ddot{w}, w^{(3)}\right) & =\frac{d}{d t}(f(w, \dot{w}, \ddot{w})) \\
& -\alpha\left(w^{(3)}-f(w, \dot{w}, \ddot{w})\right)
\end{aligned}
$$

Naturally, here we assume that $f$ is a differentiable function. Obviously, (43) is equivalent to (40). By using standard change of variables $w_{1}=w, w_{2}=\dot{u}$, $w_{3}=\ddot{w}, w_{4}=w^{(3)}$, we can rewrite (42) as

$$
\left.\begin{array}{c}
\dot{w}_{1}=\dot{w}_{2} \\
\dot{w}_{2}=w_{3} \\
\dot{w}_{3}=w_{4} \\
\dot{w}_{4}:=F\left(w_{1}, w_{2}, w_{3}, w_{4}\right)
\end{array}\right\}
$$

which has the form of (9) for $n=4$. Obviously this procedure can be extended to arbitrary dimension, provided that $f$ is sufficiently smooth.

The same approach could be generalized to discrete time case as well. Assume that the model chaotic system is given by (10) and consider the following system :

$$
\left.\begin{array}{c}
w_{1}(k+1)=w_{2}(k) \\
w_{2}(k+1)=w_{3}(k) \\
\vdots \\
w_{n-1}(k+1)=w_{n}(k) \\
w_{n}(k+1)=f\left(w_{1}(k), w_{2}(k), \ldots, w_{n}(k)\right)+z(k) \\
z(k+1)=\rho z(k)
\end{array}\right\}
$$

where $|\rho|<1$ is an arbitrary real number. Obviously, $z(k)=\rho^{k} z(0) \rightarrow 0$ as $k \rightarrow \infty$, hence the first $n$ equations of (45) and (10) are asymptotically the same. Therefore if $(10)$ has a globally attractive chaotic attractor, so does (45). On the other hand, if (10) has only locally artractive chaotic attractor, which is structurally stable in the sense given in the 
section 4, then so does (45) provided that $|z(0)|$ is sufficiently sniall.

To transform (45) into the form (10), let us define the variahle $w_{n+1}$ as follows:

$$
w_{n+1}(k)=: f\left(w_{1}(k), w_{2}(k), \ldots, w_{n}(k)\right)+z(k) .
$$

Hence, from (45) we have $w_{n}(k+1)=w_{n+1}(k)$. By using (45) and (46) we obtain the following:

$$
\left.\begin{array}{c}
w_{1}(k+1)=w_{2}(k) \\
w_{2}(k+1)=w_{3}(k) \\
\vdots \\
w_{n n}(k+1)=w_{n+1}(k) \\
w_{n+1}(k+1)=F\left(w_{1}(k), w_{2}(k), \ldots, w_{n+1}(k)\right)
\end{array}\right\}
$$

where $F$ is given as :

$$
\begin{aligned}
F(w(k))= & f\left(w_{2}(k), \ldots, w_{n+1}(k)\right)+\rho w_{n+1}(k) \\
& -\rho f\left(w_{1}(k), w_{2}(k), \ldots, w_{n}(k)\right) .
\end{aligned}
$$

\section{Conclusion}

In this paper, we considered a model-based approach to the anticontrol of some linear, time invariant systems. We considered both the continuous and the discrete time cases. Our aim was to generate a chaotic behaviour which is determined by a chaotic model, by means of an appropriate control input. To achieve this task, we assumed the existence of a refcrerice model in an appropriate form which exhibits chrotic behaviour. Then we determined an appropriate control input to match the dynamics of the systern to be controlled with that of the model chaotic system. We proved that : $i$; any controllable linear time-invariant system can be chaotified with an appropriate input, $i i$ : this approach could be generalized to a class of nonlinear systems. We proposed a simple procedure to generate such chaotic models in arbitrary dimension. We also considered the computability of the required feedback law by using only the available signals. To estimate the states of the system to be controlled, we proposed a synchronization scheme.

\section{References}

(1) Brandt, M.E., \& Chen, G., "Bifurcation control of two uonlinear models of cardiac activity," IEEE Trans. Circuits Syst. 1. 44, pp. 1031-1034, 1997.

[2] Chen, G.: \& Moiola, J.L. "An overview of bifurcation, chaos, and nonlinear dynarrics in nonlin- ear systems," J. Franklin Inst., 331B, pp. 819-858, 1.994.

[3] Chen, G. "Control and synchronization of chaotic systems, (a bibliography)", ftp.egr.uh.edu/pub/TeX/chaos.tex loginname: anonymous, password: your e-mail address.

[4] Chen, G., \& Dong, X. From Chaos to Order : Methodologies, Perspectives and Applications, World Scientific, Singapore, 1998.

[5] Chen, G., \& Lai, D. "Feedback control of Lyapunov exponents for discrete-time dynamical systems," Int. J. Bifurcation and Chaos, 6, pp. 13411349, 1996.

[6] Chen, G., \& Lai, D. "Feedback anticontrol of discrete chaos," Int. J. Bifurcation and Chaos, 8, pp. 1585-1590, 1998.

[7] Ditto, W. L. "Applications of chaos in biology and medicine," in Chaos and the Changing Nature of Science and Medicine : An Introduction, ed. Herbert, D.E. (AIP Press, NY), pp. 175-201, 1996.

[8] Fradkov, A.L., \& Pogromsky, A.Y. Introduction to control of oscillations and chaos, World Scientific, Singapore, 1998.

[9] Fradkov, A.L., \& Evans, R.J. "Control of chaos Survey 1997-2000," Proceedings of IFAC 2002 World Congress, July، 2002, Barcelona, Spain.

[10] Genesio, R., \& Tesi, A., "Harmonic balance methods for the analysis of chaotic dynamics in nonlinear systems," Automatica, 28, pp. 531-548, 1992.

11] Kailath, T. Linear Systems, Prentice-Hall, N.J), 1980.

[12] Morgül, Ö., \& Solak, E. "On the observer based synchronization of chaotic systems", Physical Review E, vol. 54, no. 5, pp. 4803-4811, 1996.

[13] Morgül, Ö., \& Feki, M. "Synchronization of chaotic systems by using occasional coupling," Physical Review E, 55, pp. 5004-5010, 1997.

[14] Morgül, Ö. "On the control of some chaotic systems by using dither," Physics Letters A, 262, pp. 144-151, 1999.

[15] Ott, E., Grebogi, C., Yorke, J.A. "Controlling Chaos," Phys. Rev. Lett., 64. pp. 1196-1199, 1990.

[16] Schiff, S.J., Jerger, K., Duang, D.H., Chang, T., Spano, M.L., \& Ditto, W.L. "Controlling chaos in the brain," Nature, 370, pp. 615-620, 1994.

[17] Ushio, T. "Synthesis of synchronized chaotic systems based on observers," Int. J. of Bifurcation and Chaos, vol. 9, no. 3, pp. 541-546,1999.

[18] Wang, X.F., \& Chen, G. "Chaotifying a stable LTI system by tiny feedback control," IEEE Trans. Circuits Syst. 1, 47, pp. 410-415, 2000. 